\title{
La educación popular católica en España: de la caridad y la asistencia al compromiso social (1953-1967)
}

\author{
Popular Catholic education in Spain: From charity and assistance to social \\ commitment (1953-1967)
}

\section{L'éducation populaire catholique en Espagne: de la charité et l'assistance au compromis social (1953-1967)}

\author{
Pere Fullana Puigserver \\ Universitat de les Illes Balears
}

\begin{abstract}
RESUMEN
La educación popular católica aparece asociada a la propaganda católica como neutralizadora de la escuela laica, como un modelo de socialización y de moralización de las clases populares en el marco del movimiento católico. El primer franquismo cedió a la Iglesia el control sobre la educación. La escuela católica de las décadas de 1950 y 1960 aparece vinculada a las clases medias y sostiene una presencia notable en la escuela primaria gratuita. A partir de 1945, sectores diversos del catolicismo optaron por intensificar su inmersión social y la educación popular crecía al amparo de aquel compromiso. La educación será una pieza fundamental en un modelo pastoral cada vez más social y eficaz. Las prácticas educativas populares del catolicismo pasan de la caridad y el asistencialismo al terreno del compromiso social, con el objetivo de llegar a los más débiles y alejados. Estas prácticas tienen también un rostro social que se refleja en nuevos compromisos en educación especializada, ocio, emancipación de la mujer y formación de adultos.
\end{abstract}

Palabras clave: Historia religiosa contemporánea. Franquismo. Movimiento católico. Historia de la educación. Educación social.

\begin{abstract}
Popular Catholic education is associated with Catholic propaganda as a neutralizer of the secular school, as a model of socialization and moralization of the popular classes within the framework of the Catholic movement. The first Franco regime gave the Church control over education. The Catholic school of the 1950s and 1960s appears associated with the middle classes, and holds a notable presence in free primary school. Beginning in 1945, different sectors of Catholicism chose to intensify their social immersion and popular education grew under that commitment. Education will be a fundamental piece in an increasingly social and effective pastoral model. The popular educational practices of Catholicism pass from charity and welfare to the terrain of social commitment, with the aim of reaching the weakest and most distant. These practices have

Date of submission: 2017-10-02

Date of acceptance: 2017-10-22

ISSN 2560-8371

http://doi.dx.org/10.24908/eoe-ese-rse.v18i0.6964

(C) Encounters in Theory and History of Education 78
\end{abstract}


a social face that is reflected in new commitments in specialized education, leisure, emancipation of women and adult education.

Keywords: Contemporary religious history. Francoism. Catholic movement. Education history. Social education.

RÉSUMÉ

L'éducation catholique populaire est associée à la propagande catholique en tant que neutralisant de l'école séculière, comme modèle de socialisation et de moralisation des classes populaires dans le cadre du mouvement catholique. Le premier régime franquiste a confié à l'Église le contrôle de l'éducation. L'école catholique des années 1950 et 1960 semble associée à la classe moyenne et occupe une place remarquable dans l'école primaire gratuite. À partir de 1945, différents secteurs du catholicisme ont choisi d'intensifier leur immersion sociale et l'éducation populaire a progressé dans cet engagement. L'éducation sera une pièce fondamentale dans un modèle pastoral de plus en plus social et efficace. Les pratiques éducatives populaires du catholicisme passent de la charité et du bien-être au terrain de l'engagement social, dans le but d'atteindre les plus faibles et les plus éloignés. Ces pratiques ont un visage social qui se reflète dans de nouveaux engagements dans l'éducation spécialisée, les loisirs, l'émancipation de la femme et l'éducation des adultes.

Mots-clés: L'histoire religieuse contemporaine. Le franquisme. Mouvement catholique. Histoire de l'éducation. L'éducation sociale.

\section{Introducción}

En el marco de la contemporaneidad la educación popular católica era una pieza fundamental del engranaje confesional, como dique de contención al liberalismo, la secularización y la influencia social de las clases emergentes. Las principales iniciativas de educación social católicas se movían inicialmente en el horizonte del paternalismo y del asistencialismo social, y obtuvieron su mayoría de edad en el contexto del catolicismo social, más concretamente encajadas en la sección de la propaganda católica, tal como se había formulado en el Movimiento Católico. El apogeo de este modelo se sitúa a grandes rasgos desde 1868 hasta mediados de la década de 1940, al hilo del espíritu de los Congresos Católicos. Pudo haber excepciones, pero en líneas generales la educación de los más desfavorecidos y de la clase trabajadora (obreros urbanos y jornaleros rurales) estaba vinculada a la cultura de la beneficencia, dependía de un proyecto de base fundamentalmente asistencial, caritativo y propagandista (Montero, 1988) y encajaba en un modelo económico dominado por el clientelismo y el caciquismo, con un peso relativamente escaso de la clase media.

Desde 1945, en especial como consecuencia de las transformaciones económicas y sociales en España, sistematizado en el Plan de Estabilización Económica de 1959, asistimos al despertar de una nueva generación que aspira a convertir la acción social católica en instrumento de transformación social y en factor de cambio cívico y político. Actores relevantes de la educación y la acción pasaron a liderar la nueva pastoral, críticos con el modelo de cristiandad, convirtiéndose en sujetos contrahegemónicos, en la medida que cuestionaban, parcial o totalmente, las estructuras sociales, económicas y políticas que sustentaban el Estado y los esquemas de poder de la Iglesia, anclados en el paradigma medieval (Kung, 2017). La educación popular crece en el ambiente, cristaliza en muchas parroquias de la periferia urbana, cuenta con 
un número relevante de centros educativos de las congregaciones religiosas, muchas de las cuales ampliaron su radio pastoral en barrios y zonas populares de las grandes ciudades, $\mathrm{y}$ madura en el marco de los movimientos especializados de Acción Católica (AC), especialmente juveniles, y colectivos cristianos diversos que, a partir de la década de 1960, apostaron de forma natural por nuevos modelos pedagógicos y nuevas opciones sociales y políticas.

Las parroquias, colegios y organizaciones laicales que se abrieron de forma natural a los medios populares se dejaron moldear al hilo de la vida y los hechos, en el paisaje de una sociedad que no era insensible a una realidad social cruda y descarnada. Una sociedad con una mayoría de perdedores, unos porque habían sido derrotados en la guerra y otros porque perdían la fe en un sistema de aspiraciones teocráticas que se mostraba vacío, superficial y retórico. Los pioneros en este despertar fueron precisamente líderes que venían de los colectivos cristianos más comprometidos durante la República y la Guerra Civil como la Juventud Obrera Católica (JOC), la Federació de Joves Cristians de Catalunya (FJCC), las Congregaciones Marianas y algunos pocos más, cuyo compromiso cristiano les había acompañado a la frontera del inconformismo.

El movimiento social católico surgido del contexto de los cincuenta se manifestaba poliédrico y se consolidaba en muchos casos como una alternativa capaz de sumarse a proyectos sociales y políticos comprometidos con el cambio (Montero, 2001; Townson, 2009). La acción social católica en España debe entenderse en el marco del paradigma social europeo a partir de 1945, con un protagonista incuestionable de la cultura democristiana que, como reivindicaba Tony Judt desempeñó "el papel principal en el establecimiento y gestión de las instituciones centrales del activista Estado del Bienestar" (Judt, 2008, p. 21).

El compromiso social permitía a la Iglesia española redimirse parcialmente de su pasado y apostar por un mayor compromiso temporal. El Concilio Vaticano II legitimaba claramente un nuevo modelo de espiritualidad desde abajo. Un compromiso en afinidad con el socialcristianismo europeo y con una cierta empatía con la socialdemocracia, pero también en sintonía con la apertura que la Iglesia española experimentaba gracias a su presencia en el Tercer Mundo y el impacto del primer eco de la Teología Popular (Preisweck, 1994; Montero, 2016). La educación popular crece a la sombra y no siempre con una gran visibilidad, al amparo de los proyectos sociales del Secretariado de Caridad, unas iniciativas que explosionarán de forma clara durante los cuarenta y cincuenta con la Ayuda Americana (Barciela, 2000) que legitimó la estructura social de la iglesia española. Un cambio que continuó con los planes de construcción de viviendas sociales, en muchos casos a la sombra de proyectos social católicos, y se consolidó con la estrategia político-pastoral de crear un nuevo tejido parroquial en la periferia de las grandes ciudades del estado. En los sesenta se había instalado la imagen de que la Iglesia española había optado preferentemente por la enseñanza secundaria y formar a los hijos de la burguesía (Hermet, 1980, p. 183).

\section{El marco de interpretación de la educación popular}

La educación popular se confunde y se difumina a menudo en el conjunto de la acción de los católicos. Aparece asociada a la escuela dirigida a las clases pudientes bajo el epígrafe de "escuelas gratuitas". Obviamente estamos hablando de aquellas propuestas del Movimiento

1 En el primer tercio del siglo XX esta tipología de escuela comprendía escuelas parroquiales, organizaciones católicas destinadas a la enseñanza, congregaciones religiosas, círculos, patronatos o asilos, integradas la mayoría en la sección de obra caritativa (Galí, 1986, p. 385-386). Poco más del 50\% de los niños inscritos en las escuelas primarias católicas durante el curso 1951-52 se beneficiaban de la gratuidad total (Hermet, 1980, p. 185). 
Católico que se caracterizan por su compromiso social, si bien para dicho catolicismo toda la educación religiosa encajaba en la estrategia antiliberal y moral que la Iglesia sostuvo durante buena parte del siglo XIX y el primer tercio del siglo XX. El presente trabajo tiene la pretensión de profundizar y contextualizar el protagonismo de la educación en la evolución del movimiento católico (Montero, 2017), en la línea que hemos venido explorando (Fullana \& Ostolaza, 2007). La reciente publicación de la monografía Espacios e intersecciones: religión y educación en escenarios canadienses y europeos, actualiza el debate y el estado de la cuestión de una temática que en España demanda con urgencia una mayor atención y posiblemente un nuevo relato ${ }^{2}$.

Desde mediados del siglo XIX, la educación popular se expresa asociada y relacionada con la necesidad de reducir el analfabetismo y corregir los déficits culturales, de una forma especial con las clases obreras, y aparece como apoyo a la incorporación de adultos, mujeres y niños hijos de la clase obrera que el propio sistema escolar entiende que no pueden quedar excluidos. Es en las ciudades donde es especialmente exigente definir el compromiso educativo y donde se explicita con mayor intensidad el compromiso confesional. Pero no fue una práctica exclusivamente urbana relacionada con el proceso de cambio y de modernización. Los sectores populares católicos generaron líneas de compromiso rural, conscientes del peso que mantenía en el conjunto del territorio español. Su puesta en escena está directamente relacionada con el proceso de consolidación del sistema educativo español a partir de 1857 y en gran medida como consecuencia del déficit de una oferta escolar pública durante la segunda mitad del siglo XIX.

Los Congresos Católicos insistieron en promover la educación y la instrucción del pueblo. Una educación relacionada con la propaganda católica, fundamentada en el catecismo, socializadora de maestros católicos, creadora de protectorado de la niñez escolar, y una escuela con un planteamiento social y preventivo que se reconocía en el rostro de Don Bosco ${ }^{3}$. En este contexto muchas congregaciones femeninas se acercaron a las jóvenes sirvientas, chicas desescolarizadas o imaginaron la oportunidad de redimir aquellas jóvenes atrapadas en el vicio y la prostitución, todas necesitaban formación, moralización y redención. Muchas de las instituciones eclesiásticas se sienten especialmente cómodas en proyectos formativos de prevención y de redención, no tanto como resultado de una lectura social, sino moral y en muchos casos únicamente religiosa.

En 1892, en el Tercer Congreso Católico, la Iglesia seguía priorizando su misión de neutralizar la escuela laica o neutra, tal como se manifestaba en la conclusión dedicada a los Asuntos de Propaganda. De tal manera que la educación católica del pueblo (escuelas nocturnas y dominicales, sobre todo) se consideraba uno de los instrumentos básicos de la superación armónica de la 'cuestión social' (Montero, 1988, p. 271).

El espíritu de la educación popular "debe trascender más allá de la esfera oficial y surgir de una iniciativa, de una acción promovida y gestionada de forma autónoma por los diferentes colectivos sociales que nutren la estructura social de una comunidad" (López Noguero, 2004, p. 7). La religión también es popular en España, una religiosidad acomodada en lo emocional y lo cultual, con un bajo perfil en todo lo concerniente a formación y de escaso peso nocional. En la Europa de las revoluciones liberales existía un cierto consenso que el liberalismo monopolizaba el discurso educativo, pero las administraciones no podían consolidar sus proyectos educativos sin contar con la Iglesia. Hasta 1870, la Iglesia había representado la única estructura sólida de control social, a partir de este momento la base de la sociedad está despertando y de hecho las

${ }^{2}$ Historia de la Educación. Revista Interuniversitaria, 35, 2016.

3 Durante la década de 1960, el catolicismo más comprometido seguía reivindicando la figura de Don Bosco. En 1961, la revista Ecclesia (p.1216) comentaba la obra Pedagogía social de Don Bosco, de Rodolfo Fierro. 
utopías sociales llevan asociados proyectos educativos y convicciones que la burguesía y la Iglesia contemplan con evidente desconfianza. Ya en 1892, la Iglesia no podía perder la batalla y consideraba seriamente que con el Sufragio Universal las clases populares estaban asumiendo su rol en las sociedades liberales, en una arquitectura y un tejido institucional y cívico que se sustentaba en la educación, con un sistema escolar en estado precario.

Por su parte, la educación popular católica aparece entre las vanguardias de la dimensión política de la Iglesia; el ámbito desde donde se transmite y se reproduce lo constitutivo de los principios religiosos, el discurso y las prácticas que constituyen el activo de la institución eclesiástica. En cualquier caso en la Europa del último tercio del siglo XIX las congregaciones femeninas masculinas francesas introducen un nivel de profesionalización y de competencia muy superior a las congregaciones meridionales. Su llegada a la Península Ibérica, a mediados del XIX, beneficia el tejido social y educativo del País Vasco, Cataluña, Madrid y las Baleares (Ostolaza, 2000; Yetano, 1988; Fullana \& Ostolaza, 2007; Dávila, 2011). En el plano masculino además hay que contar con las italianas (sobre todo los Salesianos) y la legitimidad de los colegios de la Compañía de Jesús, Agustinos y Escolapios, entre otros. Existe una red de colegios privados de corte confesional que fue perdiendo presencia a comienzos del siglo XX en beneficio de las Congregaciones, mientras que las parroquias y las congregaciones locales tienden a llenar el espacio de la educación popular básica, especialmente de las niñas. Hasta la posguerra, las parroquias no volverán a aparecer con fuerza generando colegios y centros de secundaria. Los Salesianos, además, introducen el concepto de formación profesional, de una gran relevancia en el terreno laboral. Un modelo que adquirirá dimensiones nuevas en la España de los cuarenta y los cincuenta (Prellezo, 2012).

Los agentes del catolicismo social y popular actúan en nombre de un proyecto moralizador y transformador, controlado y forjado a medida del sistema de la Restauración. Incluso Manjón, Siurot y Poveda y tantos otros pedagogos católicos como Ruiz Amado no se entienden al margen de este modelo social. Con ellos, junto a tantos otros seguramente no tan conocidos, se ponen las bases de lo que será el universo educativo de la Iglesia en España durante el siglo XX. El episcopado español de la Restauración entiende la educación en el universo de la acción redentora de la caridad, como expresaba Vicente Santiago, obispo de Santiago, en 1888 (Prellezo, 2012, p. 68).

Las prácticas de los agentes social católicos en el periodo que transcurre a partir del final de la Segunda Guerra Mundial y hasta la publicación de los Decretos Conciliares sobre educación católica cambia respecto al pasado, ponen de manifiesto la apertura de la Iglesia de base a una nueva dimensión social (el Secretariado Social de Acción Católica, representa uno de los organismos más relevantes de este nuevo proceder). Hasta el Concilio Vaticano II el catolicismo español aparece con varios discursos, por una parte una Iglesia plural en su base; jerárquica y uniforme en el vértice. La Iglesia española se había significado durante el siglo XIX y la primera mitad del XX por su sumisión a la Santa Sede, su incapacidad de desvincularse de la presión del integrismo y del carlismo y una escasa musculatura liberal. El totalitarismo de Falange no era del agrado de los obispos ni de los cuadros curiales, ni siquiera en la inmediata posguerra (Hernández, 2013, p. 233; Cañabate, 2003-2004). De hecho, el episcopado fue especialmente crítico con el control que Falange pretendía ejercer con las organizaciones de Menores, en especial con Hogares Infantiles.

Llegada la década de 1950 en España existen diversos catolicismos, en su mayoría amparados por el Régimen. Los sectores comprometidos en el campo social, rápidamente aparecen ocupando aquellas zonas grises que permiten acciones alternativas y espacios de 
oposición al sistema (huelga de tranvías de Barcelona, primeras protestas estudiantiles en la Universidad) (Raguer, 2001). En Cataluña y en el País Vasco especialmente se crearon algunas grietas en un universo aparente monolítico, por la vía del nacionalismo y por la existencia de una burguesía que permitía generar propuestas educativas, por el impacto de los desplazamientos y una generación de líderes cristianos contemporáneos al Congreso Eucarístico Internacional de Barcelona, el Concordato con la Santa Sede y los Pactos con los Estados Unidos, durante el mandato de Joaquín Ruiz-Giménez como ministro de Educación Nacional (1951-1956) (Muñoz Soros, 2016; Delgado Gómez-Escamilla, L.; Martín de la Guardia, R.; Pardo Sanz, R. (coord), 2017).

\section{La educación popular católica en un contexto generalizado de cristiandad (1868-1945)}

No cabe duda de que, en el marco del Movimiento Católico, la educación popular emerge con fuerza en 1868, en el nuevo paisaje de libertades y de eclosión de las clases populares. Ya durante el período de 1868 a 1875, el catolicismo germina con toda su fuerza. El catolicismo no puede limitarse al terreno de lo religioso, es sociedad y participa del nuevo modelo social, conjugando viejas y nuevas fórmulas educativas que nos recuerdan el modelo asistencial dominante en la cultura católica. El impacto de la obra de Fréderic Ozanam, la aparición de Don Bosco y otros educadores católicos -en el caso español Andrés Manjón será uno de los más relevantes- va a tener una gran repercusión en los ambientes populares meridionales.

Con la Restauración, en el marco del Primer Catolicismo Social, se produce un cambio relevante en el terreno educativo confesional. En el universo del movimiento católico (Montero, 2017), la educación persiste asociada a la propaganda católica, en una coyuntura, la Restauración, en la cual la escuela pública no figura todavía como el principal rival del catolicismo. Hasta 1868 la Iglesia había liderado el paisaje escolar y continuaba ejerciendo el papel de supervisión y de control a través de las Juntas Municipales / Provinciales de Instrucción Pública. En algunas regiones que hemos podido estudiar los presidentes de dichas Juntas continuaron siendo clérigos incluso en pleno Sexenio, en pleno debate sobre la secularización y la separación Iglesia-Estado ${ }^{4}$. La presión llega con las libertades (la Constitución de 1869, la Ley de Asociaciones de 1887) y el compromiso que los sectores obreristas sostienen en el campo educativo. La irrupción del asociacionismo burgués liberal o popular (casinos, ateneos, sindicatos) en la educación popular alertó a la clase dirigente católica. Las escuelas vinculadas a la Asociación de Católicos, los Círculos de Obreros Católicos y las Asociaciones de Beneficencia ejercen como vanguardia confesional en el ámbito socioeducativo. Los ejemplos relacionados con este modelo son ciertamente numerosos. El Patronato de la Juventud Obrera, fundado en Valencia en 1883 (Sánchez, 1969), puede que sea uno de los casos más paradigmáticos, entre otros motivos porque sigue en activo como centro educativo católico concertado. En líneas generales, la oferta social católica arrastra el estigma de una Iglesia nostálgica, antiliberal, vinculada al paradigma teocrático y de cristiandad.

El compromiso de los católicos con las clases más desfavorecidas tiene formatos pedagógicos de diversa consideración. Modelos que van desde el recogimiento de niños abandonados y vagabundos (Sánchez, 1969, p. 185), en este caso unido a una escuela para obreros fundada incluso antes de 1868. En otros casos se trataba de iniciativas relacionadas con

\footnotetext{
${ }^{4}$ La Junta Provincial de Instrucción Pública de las Baleares estuvo presidida en 1868 por el sacerdote y franciscano exclaustrado, Jerónimo Bibiloni Llaneras, destacado teórico del socialismo utópico y representante en Mallorca del Republicanismo Federal. La Junta Municipal de Instrucción Pública de Palma estuvo presidida por Domingo Alcina, a su vez sacerdote franciscano exclaustrado, profesor del Instituto Balear.
} 
la regeneración social (Hermanas de la Caridad); la prostitución (Adoratrices, Oblatas, Corazón de María), o la regeneración de menores (Terciarios Capuchinos), sólo por poner algunos ejemplos. El compromiso se multiplica y los modelos educativos católicos tienen a menudo un componente moral y religioso cuestionado por los poderes liberales.

Durante la Restauración la acción educativa del catolicismo fue en aumento y se consolidó hasta bien entrado el decenio de 1920, si bien muchas congregaciones a partir de este momento optaron decididamente por la escolarización reglada y su compromiso se centraba en la formación de la clase media. La reacción católica aumenta en las ciudades donde el conflicto social era particularmente vivo e intenso (Dard, 2001), especialmente durante la II República, cuando los centros y organizaciones sociales abren y ofrecen escuelas nocturnas y dominicales, con la intención de suavizar una opinión pública y un clima cada vez más tenso, que asociaba la educación católica a la burguesía y a la clase media.

No es fácil hallar excepciones en la militancia católica durante la II República y la Guerra Civil ni siquiera entre los educadores ni en las prácticas educativas, salvo algunos casos en Catalunya (Galí, 1986). La Iglesia católica española en su conjunto apoyó el golpe de estado de julio de 1936 y sus proyectos educativos y sociales no reaccionaron hasta mediados de la década de 1940 cuando se propuso extender su influencia entre las clases populares. La Hermandad Obrera de Acción Católica (HOAC), JOC, Juventud Obrera Católica Femenina (JOCF), Juventud Rural Católica (JRC), Juventud Universitaria Masculina de Acción Católica (JUMAC), Juventud Universitaria Femenina de Acción Católica (JUFAC), Vanguardias Obreras Juveniles (VOJ) y Acción Católica Obrera (ACO), por una parte; y algunas de los primeros institutos seculares femeninos y algunas congregaciones femeninas de nuevo calado se manifiestan ya a mediados de 1940 claramente comprometidos con la clase obrera y trabajan pastoral y socialmente en los barrios de la periferia de las principales ciudades españolas, especialmente en las más afectadas por el conflicto social. Conocemos el impacto de los curas obreros franceses, se ha tratado con cierta intensidad el curso de la nueva misión de los sacerdotes obreros en España (José María Llanos, Mariano Gamo, Carlos Jiménez de Parga y tantos otros) (Corrales, 2008), pero apenas se ha estudiado el protagonismo de las mujeres católicas, incluso religiosas, claramente comprometidas con la clase obrera, casos como María José Sirera Oliag (De Eguillor \& Vilanova, 2010), instituciones femeninas como el Instituto de Misioneras Seglares (IMS) y tantas otras claramente comprometidas con las nuevas pedagogías sociales, pioneras en el Secretariado de Caridad, implicadas en zonas de barraquismo y en las periferias de las ciudades con proyectos escolares, sanitarios o sirviendo en guarderías y en las primeras escuelas de Asistentes Sociales. En algunos incluso influyó claramente la figura de Simone Weil y otros iconos del feminismo católico como Dorothy Day, testimonios de la justicia social y la igualdad (Forcades, 2015). La escuela social católica femenina va ganando espacio en las publicaciones oficiosas del catolicismo español, con artículos de Mary G. de Santa Eulalia o Pilar Bellosillo y referencias a la obra de M. Laffite (1964).

No cabe duda que el primer franquismo (1939-1953/59) mantiene un vínculo estrechísimo con el catolicismo, al que ha invitado a compartir el poder y le ha ofrecido algunos de los poderes con los que más y mejor había soñado la Iglesia. La cuenta de resultados en el seno del catolicismo no había sido tan sustancioso como habían augurado los ideológicos de la cruzada, entre otros motivos porque todo se había construido sobre un nacionalcatolicismo híbrido. Su primer objetivo consistió en desmantelar el sistema educativo de la II República, construir un nuevo modelo fundamentado en la pedagogía tradicionalista, de base católicaintegrista. La práctica educativa de la posguerra, contaminada por el poder omnipresente e 
ideológico de los vencedores, entre los cuales figuraba la Iglesia en bloque, no dio los resultados deseados, porque moralmente no contaba con crédito suficiente y sirvió para aumentar el descrédito público de buena parte de la nueva clase dirigente. Un proyecto que oscila entre el modelo propositivo de una Iglesia católica herida y escasamente autocrítica, con otras propuestas exigentes, autónomas, que trataban de defender su posición y su misión al margen o enfrentada si es necesario al totalitarismo del Régimen.

El sustrato moral del modelo del nuevo Estado era débil y decepcionó a muchos católicos, aunque continuaron defendiendo el franquismo naciente. Los religiosos, atentos a los beneficios que les ofrece el régimen, ejecutan proyectos educativos de nuevas dimensiones, pero no todos se someten de forma incondicional. Ya en la inmediata posguerra, la Iglesia desconfía del modelo educativo del Régimen, el modelo totalitario ha perdido en Europa y el nuevo escenario, aparentemente leal al Régimen, permite un semillero de catolicismos marcado por el pluralismo y por deseos de libertad respecto al sistema político, a pesar del flirteo permanente con el Régimen. La práctica escolar permite a la Iglesia contratar maestros que han sido sancionados por las comisiones depuradoras y en algunos casos incluso después de pasar varios años en la cárcel (Fullana, 2005). El planteamiento de las escuelas gratuitas que ofrecían las Congregaciones se había quedado obsoleto y los propios Institutos abandonaron definitivamente dicha oferta.

\section{Los católicos ante el reto de educar una sociedad desescolarizada (1945-1967)}

A partir de 1945, el modelo de Iglesia hegemónico sería cuestionado muy rápidamente, incluso desde su propio interior. La Iglesia transformando el mundo se transforma a sí misma. Para la Iglesia apenas han cambiado los principios, todo parece estar en su sitio, pero todo está mutando a gran velocidad desde mediados de los años cincuenta. "Enseñar al que no sabe" era el título del editorial de Ecclesia el 5 de noviembre de 1960. Enseñar es una obra de misericordia, pero también comenzaba a ser una obra de justicia, un proyecto de transformación y un ideal asociado a las nuevas presencias cristianas en barrios y zonas hasta ahora abandonados. Indudablemente la educación tenía una dimensión social y política, pero no era menos cierto que estaba cambiando claramente el modelo de sociedad, una transformación que afectaba a los efectivos eclesiásticos y a un contingente de laicado, numeroso, joven y con esquemas mentales claramente diferenciados de las generaciones anteriores. La pastoral de la Iglesia se manifestaba cada vez más social y más eficaz

Entre los principales factores del cambio, con sus persistencias y resistencias, hay que destacar la aparición de una sociedad tecnocrática, preludio de lo que sería la sociedad del bienestar. En conjunto una sociedad con fragilidades evidentes, que expresaron Ivan Illich y tantos otros pedagogos católicos. Un análisis que compartía Alfonso Comín en Cuadernos para el Diálogo. Dos visiones críticas en el contexto de 1960 y que expresan parcialmente el clima del cambio. Los jóvenes católicos que no habían hecho la Guerra, que la habían padecido pero que no se identificaban con el ambiente martirial politizado de la posguerra, anhelaban cambios sustanciales desde el final de la Segunda Guerra Mundial. En los ambientes cultos, universitarios o comprometidos con el movimiento católico se observaba la realidad con optimismo. Desde la finalización de la Segunda Guerra Mundial, los movimientos especializados de AC (JOC, HOAC) introducen en la Iglesia un nuevo campo de visión, de acción y de formación que permite a sectores conscientes de la Iglesia afirmarse en una posición crítica con la institución.

\footnotetext{
${ }^{5}$ Hacia una pastoral social eficaz, Ed. Estela, 1966.
} 
Los tiempos eran diferentes a los de restauración social católica propios del primer franquismo (Montero \& Louzao, 2015), un tiempo en el cual el ardor juvenil católico se canalizó también en organizaciones y movimientos apolíticos o claramente integrados en el Régimen (Cursillos de Cristiandad), críticos con la moral dominante, disconforme con la rutina religiosa y con el conformismo del orden establecido. Estos movimientos también emergían del seno de AC, pero por regla general no se proyectaban al terreno social y persistían en el plano del espiritualismo tradicional. Muchos católicos seguían colaborando con el plan de formación de Falange, del Frente de Juventudes o la Sección Femenina, donde habían adquirido una cierta experiencia. En la escuela pública, en las instituciones públicas de acción social (penitenciarias, beneficencia o sanitarias), en las organizaciones juveniles y en tantos otros espacios de formación se estaban viviendo experiencias de un gran interés para el futuro de la Iglesia. Un catálogo notable de revistas (Germinabit, El Ciervo, Cuadernos para el Diálogo) y editoriales (Spes, Atlantida, Estela) católicas aparecen en el escenario, traduciendo y publicando obras que introducen a la militancia católica hacia horizontes sociales nuevos. La Iglesia y la escuela aparecen entre los principales focos de irradiación de valores que cuestionaban la España reaccionaria, empobrecida culturalmente. En estos ámbitos se estaba produciendo una oscura introspección (Amat, 2017, p. 37). Ya en la década de 1950 asoman las primeras divergencias de los sectores populares católicos con el franquismo y con la institución eclesiástica (Montero \& Louzao, 2016).

La educación popular aparece como un ámbito de diálogo entre el nuevo clero y nuevos docentes. Un encuentro que se comparte a la sombra de una Iglesia en la que gana terreno la diversidad y el pluralismo a pequeña escala. La acción en la periferia de las grandes ciudades (Madrid, Barcelona, Valencia, Sevilla), la emigración interior y la acción social cristiana, hacen posible una cierta conversión del catolicismo por la vía de la acción social. Inclusive en estos ambientes populares, alejados del modelo de confort burgués, la parroquia y el compromiso de los católicos cobra un sentido nuevo. Las grandes ciudades se urbanizaron alrededor de un mapa parroquial, integrado y acompañado por colegios confesionales, centros asistenciales, e infraestructuras de naturaleza diversa (hogares para mayores, guarderías, dispensarios y centros sociales). La educación y la formación ciudadana tuvieron un rol de primer orden en la acción misionera en los barrios obreros ${ }^{6}$. Las iniciativas juveniles y el compromiso de los católicos en dichos barrios originaron un sinfín de iniciativas muchas de ellas a cubierta de la ideología y la mentalidad asistencial (Misioneras de Jesús Obrero (1955), Misioneros de la Esperanza en 1956 (MIES)). En las élites y entre los laicos cristianos comprometidos en el universo universitario la situación era diferente. Jaime Vicens Vives a comienzos de los sesenta recomendaba a Josep Benet el libro Les prêtes ouvriers (Amat, 2017). Los congregantes universitarios, cercanos a la Compañía de Jesús, colaboraron con el P. Llanos y el P. Díez Alegría en el Pozo del Tío Raimundo, hoy icono y referencia de las experiencias del cristianismo popular de los años cincuenta (Rodríguez, 2007, 2017; Lamet, 2013).

De los seminarios españoles salen un clero joven, de una manera especial a partir de 1945, algo independiente, incluso seminaristas provenientes de familias de vencidos, hijos del silencio y del miedo. Las congregaciones religiosas trabajan pastoralmente en la periferia de las ciudades, en muchos casos regidas por religiosos que han vivido la República y la Guerra y se habían entregado en brazos del nuevo régimen. Es más, en muchos casos la vida religiosa de los cuarenta que se manifiesta más tradicional que el clero secular, a raíz de su incardinación en barrios obreros y su inserción en zonas populares, ayuda a tomar consciencia de que necesita rehabilitarse, reconciliarse con los marginados y desfavorecidos. La vía social se convierte en un

\footnotetext{
${ }^{6}$ Acción misionera en el barrio obrero, Ed. Estela, Barcelona 1960.
} 
camino de rehabilitación, de regeneración, de encuentro entre creyentes anticlericales y un clero comprensivo y pastoral que antepone la lectura social a la imposición retórica y dogmática. Junto a la jerarquía emerge un laicado que viaja, estudia y que se contrasta y confronta en el exterior. Los catolicismos progresistas europeos llegan a España de la mano de laicos, muchos de ellos vinculados a jóvenes democristianos que viajan y participan en encuentros internacionales (Pax Cristi, por ejemplo). La influencia de Alcide de Gasperi, Gianni Baget Bozzo o Igino Giordani es evidente durante estos años. La regeneración social llegaría en esta dirección. El modelo social centroeuropeo aterriza en España, en muchos casos a través del catolicismo francés o por la vía italiana.

Para entender el proceso de cambio en el interior de la Iglesia y su compromiso a favor de un modelo educativo alternativo es imprescindible tener en cuenta la creación de las Escuelas Normales de la Iglesia (siguiendo el plan de 1948) (Matas, 2015; Holgado \& Márquez \& Martín, 2013), la fundación de las Facultades de Pedagogía y la profesionalización de muchos religiosos dedicados a la educación. La propia institución eclesiástica, experimenta un proceso de pedagogización, de tal manera que en 1960, Juan XXIII afirmaba que el pastor de almas había de ser maestro, educador y psicólogo ${ }^{7}$, y los alejados pasaban a ser los preferidos y los elegidos al hilo de lo que se debate en muchas de las semanas parroquiales de la década.

En la base, la Iglesia vivía una coyuntura de esperanza y de una cierta euforia. Los laicos adquirían un protagonismo jamás imaginado, aunque la Iglesia era más clerical que nunca y rápidamente los obispos percibirían los peligros del desconcierto. Miguel Benzo apostaba por el testimonio seglar, no mediante el poder, sino con el testimonio en un artículo publicado en la revista Ecclesia titulado "Teología del testimonio seglar" $"$. La institución eclesiástica se apoyaba en sus propias persistencias. Como consecuencia de la apuesta tecnocrática, reforzaba su vinculación a la patronal. En 1960, se celebraba en Sevilla la VI Semana Nacional de Acción Social Patronal, donde cien empresarios españoles debatieron en torno a "la libertad y responsabilidad en la vida social". Se insistía en la actitud cristiana ante los problemas morales de la estabilización y el desarrollo económico ${ }^{9}$.

No cabe duda que la Iglesia española recibía un trato de favor y una "protección" del Estado, que se reflejaba en ventajas en el campo educativo. La "protección" conllevaba una cierta libertad de movimiento lo que derivó en cambios en la base de la Iglesia al producirse un acercamiento al sector obrero (JOC, HOAC) y a sectores alejados y periféricos, en algunos casos con el apoyo de colectivos de avanzada provenientes de ambientes burgueses, como las VOJ. Las células cristianas tienen una presencia notable en ambientes desfavorecidos, en algunos casos la Iglesia fue la única institución comprometida en los barrios de barracas ${ }^{10}$, entre otros compromisos construyendo escuelas. Los agentes cristianos que intervienen en este proceso están vinculados a las congregaciones religiosas masculinas y femeninas, con sus organizaciones juveniles en la vanguardia (De Andrés, 2013) creando centros de jóvenes y espacios de desarrollo comunitario, germen del primer movimiento vecinal organizado (Townson, 2009).

\footnotetext{
7 Juan XXIII, A los Cuaresmeros de Roma, Ecclesia 1960, 293-294.

8 Ecclesia 1960, 253-254.

9 Declaración colectiva de los Metropolitanos Españoles, enero 1960.

10 “'L’Església era la única institució que actuava socialment en els barris de barraques. En el cas de Can Valero, un capellà carmelita, el pare José Miguel, es va encarregar als anys cinquanta d'anar dotant el barri de les infraestructures asistencials mínimes [...] va fer possible la creació d'una petita escola i la posada en marxa d'un dispensari sanitari [...] A principis del seixanta, les Teresianes del Niño Jesús de Praga, amb el suport del mateix constructor, edificaren una escola a las Banderas" (Grup, 2008).
} 
Los movimientos de renovación pedagógica en algunos casos también tuvieron su punto de partida en círculos católicos, no siempre directamente en sintonía con la jerarquía. Es el caso de Cataluña con un movimiento de inspiración cristiana ya en sus orígenes (Monés, 1981). El Institut Catòlic d'Estudis Socials de Barcelona (ICEBS) se había creado en 1951. En la misma línea actuaron diversos colectivos como el Centre d'Influència Catòlica Femenina (CICF), la Institució Pedagògica Rosa Sensat, el Centre d'Estudis Francesc Eiximenis y otros como el Moviment Escolta, o individuos como Frederic Bassó (consiliario de la escuela Talitha ya en 1956-57). Algunos grupos católicos, entre 1955 y 1977, elaboraron un pensamiento crítico y estimularon una línea de compromiso crítico con la escuela tradicional nacionalcatólica, de tal manera que durante los sesenta la infantería social de la Iglesia evolucionó desde propuestas asistenciales hasta convertirse en introductores del desarrollo comunitario (Grup, 2008, p. 179).

\subsection{Sectores relevantes del catolicismo se acercan a las comunidades populares}

Si se analizan con rigor algunas de las acciones de animación socioeducativa católica ya en los cincuenta, se puede confirmar la existencia de cambios antes del cambio. Antes del Concilio Vaticano II se ponen las raíces del cambio, con testimonios y prácticas muy concretas, tal como han destacado antropólogos, urbanistas, historiadores y sociólogos (Grup, 2008; De Andrés, 2013; Rodríguez 2017). Algunos pocos reconocen ya la mano de los católicos en la transformación de los sesenta, un peso que la historiografía debería rastrear en otros ámbitos de las ciencias sociales, base que puede explicar los cambios en el subsistema educativo católico, que les permite flexibilidad, dinamismo y diversificación (Viñao, 2004, p. 203-210).

Ya a comienzos de la década de 1950, sectores relevantes de la Iglesia perciben que la Iglesia se debe a la comunidad (Raguer, 2006). Grupos cristianos que no quieren ser utilizados ni por unos ni por otros, quieren ganarse su propia credibilidad en el compromiso y el testimonio social. Desde HOAC y JOC se ofrecieron cuadros preparados y formados para actuar en los ambientes más estridentes socialmente (Sanz, 2001; Dard, 2001). No cabe duda, por otra parte, de que durante los sesenta no todos los agentes cristianos son antifranquistas, pero se cuestiona la tesis de la identificación absoluta entre el factor católico y el factor nacional, y comienzan a manifestarse fisuras en el nacionalcatolicismo (Roig, 2002, p. 121). Parte de estas grietas eran resultado de la evolución de la Acción Católica, de sus movimientos especializados y de los colectivos laicales de los institutos religiosos.

La mayoría de estos colectivos evolucionan en contacto pastoral-social con comunidades populares (periferia urbana, colectivos rurales reivindicativos, inmigrantes, mujeres, jóvenes, etc.) y crecen al amparo de pedagogías que parten del ambiente ${ }^{11}$. La planificación y ejecución de los "poblados dirigidos" de Madrid y de otros ciudades, con la construcción de barrios de viviendas, genera intervenciones educativas relevantes y aparecen iniciativas y figuras de educadores que parten de la realidad, la analizan en profundidad y actúan en consecuencia. Esta metodología proveniente del método de revisión de vida se sostuvo en gran parte con el apoyo de la aplicación de la sociología, con iniciativas de calado, entre las cuales despuntaron el P. Jesús María Vázquez (promotor de la revista Barriada y vida, dedicada a la sociología) y Rogelio Duocastella con la creación del Centro de Estudios de Sociología Aplicada (Carmona, 2016, p. 65-69). Esta lectura social de la realidad cuenta con apoyos en la jerarquía (el Dr. Ricote, obispo auxiliar de la diócesis de Madrid, favorecía en Madrid la obra de los docentes en los suburbios) $)^{12}$, un deseo y una exigencia que se hace extensiva a las parroquias, comprometidas

\footnotetext{
${ }^{11}$ Ecclesia 1960, 141-142

12 Ecclesia 1960, 731-732.
} 
con la educación popular. Para los obispos, la parroquia debía ser el centro y desde esta mediación debía gestionarse toda la acción de los católicos. Esta percepción se hace también cada vez más extensiva a las órdenes y congregaciones religiosas, en especial a los Escolapios, Jesuitas, Salesianos y tantos otros presentes en poblados dirigidos, barrios de barracas y en áreas periféricas de las grandes ciudades (De Andrés, 2013).

Muchos de estos agentes cristianos aumentan su relación con la educación, favorecidos por la vinculación entre el catolicismo y bienestar social en la España de los 50 (Montero, 2007, p. 112-119). Como es obvio, las congregaciones religiosas multiplicaron su red de centros educativos en todo el territorio. Entre todas estas iniciativas, no cabe duda de que su presencia en los ambientes populares cuestionaba profundamente el sentido de las obras educativas, en especial el de sus proyectos benéficos o gratuitos. Solo este aspecto ya merecería un tratamiento monográfico, al igual que el compromiso de la mujer creyente en las periferias, a través de tantísimas iniciativas de diverso calado, como Hogar Mater Amabilis (Vallecas, Madrid), la formación de las jóvenes en las barriadas ${ }^{13}$ y el proceso de formación y de compromiso de tantas religiosas que cursaron estudios de magisterio, pedagogía, enfermería o se graduaron como asistentas sociales durante estos años. El nivel de profesionalización de las religiosas creció de una manera notable y el contacto con las realidades sociales trasladó estas problemáticas a la agenda de las superiores mayores de muchos institutos. En 1960, llamaba la atención la graduación de 25 religiosas bachilleres laborales preparadas para trabajar en escuelas de formación profesional ${ }^{14}$. En el interior de congregaciones e institutos seculares femeninos, junto a los movimientos especializados de AC femenina se estaba gestando una nueva mentalidad, acorde con el compromiso social (Moreno, 2005, p. 109).

Estos procesos de cambio se debían a la progresiva pedagogización de la Iglesia, que incluye la catequesis (la revista Sinite, promovida por los Hermanos de las Escuelas Cristianas, da fe de ello); un mayor compromiso en los temas de la infancia, a través de la Comisión Católica de la Infancia; así como la implicación de los movimientos laicales en la educación popular (la Federación Española de Congregaciones Marianas Universitarias FECUM, entre otros) (Sainz, 1996), una suma de ámbitos que tienen en común la esperanza que una generación de jóvenes católicos depositaba en la educación como instrumento de transformación de la realidad. Este fenómeno era posible entre otros motivos gracias al protagonismo que adquiría la Universidad (en 1960, los católicos reclamaban una Facultad de Educación en la Universidad de Salamanca) y la democratización de los estudios universitarios, y se constataba un aumento de convocatoria de jornadas de estudio sobre educación expresión de la implicación de los católicos en este campos ${ }^{15}$. El nuevo paisaje educativo se ampliaba de forma exponencial durante los sesenta, en especial como consecuencia del prestigio que adquiría la escolarización y la formación, tanto para las clases populares como para las clases medias en ascenso. Para dar cobertura a esta demanda se crearon residencias para estudiantes en muchas ciudades, casi al mismo nivel que también surgieron residencias para jóvenes obreros, en muchos espacios religiosos que se adaptaban a usos nuevos, como consecuencia del cambio de paradigma social en la propia institución.

La comunidad cristiana asumía el reto de afrontar lo que se denominaba en los medios como "los grandes deberes con el pueblo", todo con la finalidad de mejorar al pueblo y llegar a todos los rincones de la diócesis. En la iglesia española inquietaba la situación de la juventud

\footnotetext{
13 Ecclesia 1960, 802.

14 Ecclesia 1960, 900.

15 Ecclesia 1960, 85: Jornadas Pedagógicas de Logroño.
} 
universitaria, pero también la juventud rural (I Congreso Mundial de la Juventud Rural), unas problemáticas que se sumaban a la urgencia de acercarse a los colectivos sociales más alejados, al tiempo que se percibía un cierto distanciamiento de sectores hasta ahora tradicionalmente practicantes. Una inquietud sobre la que reflexionaba, en 1958, el entonces pedagogo escolapio Rafael Pérez Azpeitia en su publicación Apostasías en masa.

Ya a comienzos de los sesenta en el seno de la Iglesia española existe una cierta percepción de un mayor compromiso temporal que ciertamente preocupa a los colectivos más conservadores y tradicionales. Una cierta reflexión sobre el campo de lo temporal está muy presente incluso en las publicaciones oficiosas de la jerarquía ${ }^{16}$; después de que se mostrara partidaria de equilibrar la "acción temporal en conformidad con la fe"" del lado de los sectores liberales y apostaban por el desarrollismo, como quienes aumentaban su compromiso con los sectores marginales de la sociedad venían llamados a buscar un cierto equilibrio y para ello se hablaba de la igualdad de oportunidades, y desde la revista Ecclesia, ya en 1961, se lanzaba una editorial titulada "Igualdad de oportunidades"18 donde se apostaba claramente por incrementar los presupuestos educativos, argumentando que el aumento de la renta debía tener una proyección en el terreno de la educación.

Por otra parte, en algunos editoriales de la revista Ecclesia $^{19}$ se reflexionaba sobre la necesidad de recuperar los sectores alejados de las parroquias, se constataba la baja cultura del mundo obrero y la de otros muchos colectivos sociales, y se insistía en la necesidad de la educación. Obviamente en este proceso de liberalización que caracterizaba la realidad española, el crecimiento de las infraestructuras educativas de la Iglesia fue notable durante estos años. Puede que incluso desproporcionado. En cualquier caso, también se percibía un clima de mayor exigencia en la oferta educativa, una mayor presencia de los institutos en el terreno universitario, pero también en la formación profesional y en la educación popular; una presencia que se hacía más visible, si cabe, porque muchos de los institutos religiosos contaron con grupos postescolares juveniles que se correspondía con un tiempo de gran visibilidad para la Iglesia, porque contaba con un ascendiente notable sobre la juventud y con un movimiento católico juvenil relevante desde muchos puntos de vista (Fullana \& Montero, 2003-2004).

La doctrina social de Juan XXIII legitimaba el compromiso socioeducativo de los colectivos emergentes de la Iglesia en España. La letra y el espíritu de Mater et Magistra (1961), tuvo una recepción de primer nivel entre los sectores católicos, en especial entre los más comprometidos socialmente. Estos mismos colectivos se sintieron reforzados en sus planteamientos educativos y sociales, y sus apuestas salieron fortalecidas del Concilio Vaticano II. Marta Mata analizaba en 1966 la "Declaración sobre Educación Cristiana" que exhortaba a los católicos a colaborar en la tarea de extensión y la cualificación de la educación a todo el mundo. De alguna manera el magisterio asumía una práctica y un compromiso que había calado en los ámbitos donde los cristianos convivían con la marginalidad y abandonaban el territorio de la confesionalidad. En el Capítulo IX, la Declaración admite la diversidad de escuelas católicas, según las necesidades de lugares y tiempos, y encaminaba a la Iglesia a enfocar preferentemente su acción a aquellos ámbitos con más deficiencias: los pobres, los huérfanos, los analfabetos y los no creyentes ${ }^{20}$. Para Marta Mata este punto cuestionaba profundamente la opción única para

\footnotetext{
16 "El campo de lo temporal": Editorial de Ecclesia, 15 de julio 1961: pp. 876-877.

17 Ecclesia 1961, 293.

18 Ecclesia 1961, 1004.

19 Ecclesia 1960, 35.

20 Declaración ""Gravissimum educationis "", del Concilio Vaticano II.
} 
las escuelas para ricos y las clases dirigentes donde algunas congregaciones religiosas parecían estar instaladas (Mata, 1966).

Por otra parte, las diferentes tradiciones educativas en el seno de la Iglesia española muestran su preocupación ante el nuevo protagonismo que adquiere la juventud y ante la influencia de la denominada segunda revolución técnica. En este sentido, la Iglesia se mostraba especialmente atenta ante las nuevas oportunidades que ofrecían el ocio y los medios de comunicación de masas, entre los cuales despuntaba la televisión. Desde todas las instancias se apoyaba que predicadores y educadores enseñaran que la televisión encerraba grandes esperanzas y graves preocupaciones ${ }^{21}$; unos retos que compartía con respecto al cine y la radio. De tal manera que la influencia del ambiente en la obra educativa (cine, radio, televisión, calle, familia, lectura, amistades) fue el tema de análisis elegido en 1960 por parte de la Federación Española de Religiosos de la Enseñanza (FERE) ${ }^{22}$. Se hablaba de la influencia de la prensa en la educación, todavía muy dependiente del régimen. La FERE, de hecho, invitó al director general de prensa; y la institución eclesiástica tuvo también un papel de primer orden en la puesta en funcionamiento de Teleclubs parroquiales.

En un paisaje de aparente armonía entre la Iglesia y el Estado, crecía la sensación de aumento del número de alejados de un sistema que manifestaba fisuras evidentes. Transcurridos más de diez años del final de la guerra, después de todo el compromiso institucional, la jerarquía eclesiástica se manifestaba altamente preocupada ${ }^{23}$. Por una parte, existía una inquietud evidente generada por la técnica y su influencia en la juventud ${ }^{24}$; los primeros efectos del turismo se explicitaban y aparecían los programas de ocio y turismo dirigidos al intercambio juvenil, controlado por albergues de colonias vinculados a las diócesis o a colegios católicos. De tal manera que la "educación social y cívica en una sociedad de masas", figuraba como tema central de la XXII Semana Social de España, celebrada en Oviedo en 1963.

4.2 La Iglesia española ante el reto de la educación especializada, el tiempo libre, la alfabetización de los adultos y la emancipación de la mujer

Existe un cierto consenso que después de la Guerra Civil, que el Auxilio Social y las organizaciones de corte asistencial del régimen marcaron el estilo de los centros y de las instituciones "benéficas" o de "auxilio" (derivados de una identidad nacional, católica y sindicalista) (Palacio Lis \& Ruiz Rodrigo, 2010, p. 164). El modelo público que la sociedad del bienestar estaba originando en Europa no cabía en la España de los cuarenta y cincuenta. En términos generales, los centros de protección, hogares, centros de beneficencia y reformatorios fueron utilizados como propaganda del régimen, pero en la mayoría de casos se trata de centros que sufrieron una inmersión formativa basada en el nacionalcatolicismo, entendido como un modelo fundamentado en una religiosidad pietista y un modelo de convivencia riguroso, represivo y autoritario. El castigo, la escasez de recursos, el miedo y la represión son algunas de las notas que caracterizaron la reeducación en el período de 1939 a 1960. Para ejecutar este plan el Régimen contó con la colaboración, plural desde el punto de vista emocional, de las diócesis y de las congregaciones religiosas. A partir de la década de 1960 se suavizaron los reglamentos, muchos centros se secularizaron, incorporaron profesionales formados en el mundo universitario,

\footnotetext{
21 Ecclesia 1960, 45.

22 "Los influjos del ambiente en la obra educativa", Ecclesia 1960, 55-56.

23 "Los alejados de la parroquia"(editorial), Ecclesia 1960, 35.

24 "Es necesaria la presencia de los católicos en la educación de la juventud. Mensaje del Papa en el XXX aniversario de la Divini Illud Magistri”, Ecclesia 1960, 75.
} 
se descentralizaron los centros. A su vez se generaron iniciativas nuevas, propias del nuevo espíritu pedagógico que caracterizó la época, resultado de la implicación de jóvenes educadores católicos, en gran medida provenientes del escultismo, de la AC especializada o de grupos postescolares vinculados a las congregaciones religiosas.

En la base del catolicismo español se estaba produciendo una metamorfosis imperceptible, lógico a tenor del proceso de liberalización de la sociedad, del turismo y de la onda expansiva que llegaba de las "nuevas esperanzas progresistas" europeas, generadoras de propuestas e iniciativas relacionadas con la educación especializada. Ya en 1958 se había creado la Comisión Católica Española de la Infancia ${ }^{25}$, como consecuencia de una lectura nueva de la realidad social y como fruto de una nueva mentalidad. El propio Juan XXIII, el 14 de abril de 1960, en su discurso a los participantes en el Congreso Internacional para la protección de la infancia y la adolescencia, se refería a la infancia y adolescencia inadaptada. En su parlamento contemplaba la necesidad de contar con técnicos y especialistas, y abría el futuro de estas instituciones a individuos preparados y formados ${ }^{26}$.

La propia revista Ecclesia va a prestar durante estos años una mayor atención a los servicios sociales y se convertirá en portavoz de noticias relacionadas con la educación especializada, tal como se entendía en la década de 1960. En este sentido se hacían eco de los especiales deberes de caridad con los niños enfermos; se reproducían los discursos del Papa Juan XXIII a la Asociación Italiana de las Familias de "Retrasados Mentales"27, a la Acción Católica de Sordomudos $(1960,374)$ y a un grupo de niños ciegos, del Instituto Pablo Colosino, entre otros actos que repercutían en la opinión católica del momento. El catolicismo español se hizo muy presente en el campo de la juventud inadaptada en la España franquista, un fenómeno social ya de un cierto calado, a medida que avanzaba el proceso de liberalización de la sociedad (Nuq, 2012). Las publicaciones católicas también serán el reflejo de una mayor sensibilidad con todo aquello relacionado con la Obra Protectora de Menores, de una forma especial con la dedicación de los Terciarios Capuchinos. La revista Surgan (editada desde 1949 hasta hoy por los Terciarios) se ha convertido en una de las principales fuentes para conocer el calado de estas iniciativas confesionales. Una obra que junto a tantas otras como la de los Hermanos Obreros de María (Granada) que trabajaba con niños, la mayoría hijos de reclusos, abandonados o deficientes $^{28}$ y la elección de diversas congregaciones que optaron durante los sesenta por el mundo de los discapacitados, como las Franciscanas Hijas de la Misericordia, con la creación del Centro Mater Misericordiae, en Palma (Mallorca) (Fullana, 2011, p. 353-364; Matas, 2015a).

El catolicismo no es ajeno a los síntomas de malestar que comenzaba a manifestar la sociedad española a comienzos de los sesenta. Síntomas que se habían relacionado con la represión durante la primera etapa del franquismo, con la colaboración de la Iglesia, en espacial de algunas congregaciones (Nuq, 2012), un paisaje que cambia a medida que las élites de esta misma Iglesia sintonizan con otros contextos, leen, traducen e introducen nuevas metodologías pedagógicas en el campo de la educación especializada, en especial en reformatorios y centros de reclusión de adolescentes y jóvenes, donde el ambiente había sido oscurantista y poco alentador (Regas, 2004; Armengou \& Belis, 2016). La propia revista Ecclesia publicita la obra de Burns

\footnotetext{
${ }^{25}$ Ecclesia 1960, 886-888. https://www.mecd.gob.es/dctm/revista-de-educacion/1962144/1962re144estudios02.pdf?documentId=0901e72b8186383a

${ }^{26}$ Ecclesia 1960, 587-588.

${ }^{27}$ Ecclesia 1960, 329.

$28 \mathrm{http} / / /$ www.fundacion-hom.org/wp-content/uploads/2016/05/REVISTA-HNO.-CARLOS-1.pdf
} 
dedicada a los niños inadaptados ${ }^{29}$, reproducía el discurso que Juan XXIII había ofrecido en el V Congreso de la Asociación Internacional de la Juventud inadaptada y se hacía eco del coloquio sobre la inadaptación juvenil desde el punto de vista religioso.

Desde la base de la comunidad eclesial, en especial por parte de los efectivos más jóvenes del estamento clerical y de los jóvenes laicos que viven en primera persona los nuevos tiempos de apertura y liberalización de la sociedad, existe una cierta conciencia de que la gestión del tiempo libre y el ocio de adolescentes y jóvenes es fundamental en los procesos de formación. La sociedad en pleno vivía plenamente la denominada edad del ocio, en la cual la educación no es exclusiva de la industria escolar (Illich, 1971). En buena medida el catolicismo más sensible tiene la mirada puesta en Francia y en su universo de ofertas de tiempo libre, en este sentido se mantienen contactos con el Movimiento coeurs vaillants, de Francia; se apuesta por el movimiento scout que acababa de crear, en 1961, la Oficina de Información y Coordinación del Escultismo Católico, con participación de Barcelona, Valencia, Palma de Mallorca, Madrid, San Sebastián y Bilbao, y se implementa la presencia católica en la educación de la juventud, con ofertas de espacios de colonias, en muchos casos vinculadas a Caritas. La convocatoria de los Juegos Olímpicos de Roma 1960, había provocado un cierto despertar del catolicismo que sigue los mensajes renovadores y en algunos casos inclusos sorprendentes de Juan XXIII. Desde el punto de vista de la educación social, vale la pena recordar como el universo católico incorpora conceptos como la energía y virtud necesaria al cristiano, ahora vinculado al deporte. En este momento, llama la atención el discurso de Juan XXIII dirigido a "los inválidos"30 participantes en los juegos internaciones, considerados los primeros juegos paralímpicos de la historia, con la participación de 209 deportistas (ninguno español).

Desde los sectores confesionales se seguía con interés la programación de la Comisaria de Extensión Cultural (Vilanou \& Planella, 2010, p. 172), creada por decreto de 18 de diciembre de 1953, con el objetivo de difundir los valores de la cultura entre todos los españoles en edad postescolar o educación de adultos. La Comisión trabajó con la Junta de Analfabetismo en las denominadas "misiones culturales". La Sección Femenina creó las "Cátedras ambulantes" dedicadas a educar e incorporar a la mujer en la vida ciudadana. El Ministerio de Información y Turismo promovió también iniciativas de carácter formativo (Vilanou \& Planella, 2010, p. 173). A medio camino entre el mundo juvenil y el de los adultos, el laicado católico y las asociaciones vinculadas a la Iglesia, en especial aquellas más comprometidas directamente con los sectores populares, están atentas a las nuevas necesidades. En el entorno de parroquias, institutos y asociaciones se despierta una cierta sensibilidad con las problemáticas relacionadas con la mujer, en especial con las más jóvenes. En este sentido se crearon residencias y comedores sociales para chicas desplazadas ${ }^{31}$; se implementaron obras destinadas a la redención de mujeres, (Villa Teresita, creada en 1943, rápidamente contaba con cuatro casas en ciudades españolas) y las congregaciones que tenían este carisma (Adoratrices y Oblatas del Santísimo Redentor) refuerzan su presencia en los ámbitos populares y abandonan lentamente reformatorios y centros educativos de corrección tradicional. Juan XXIII hablaba en 1960 de la protección a la joven, en un mundo hostil ${ }^{32}$. En España, la Obra Social Femenina inauguraba, en 1961, una casa en Murcia; y las Siervas de las Obreras o Misioneras de María Inmaculada obtuvieron la aprobación

\footnotetext{
${ }^{29}$ Ch. BURNS, Los niños inadaptados, Herder, 1960, publicitada en Ecclesia 1960, 870.

30 Hoy nos parece políticamente incorrecto el lenguaje, pero éste era el propio de 1960 y así aparecía en el titular de Ecclesia y seguramente en el de los medios de comunicación en general.

31 http://www.villateresita.org/index.php/quienes-somos/nuestra-historia

32 Discurso de Su Santidad a la obra de Protección de la Joven: Ecclesia, 1960, 1613-1614.
} 
diocesana en 1961. Este nuevo marco de compromiso en el terreno de la formación y el acompañamiento de las jóvenes estaba directamente relacionado con el cambio de paradigma en todo lo concerniente al trabajo de la mujer, encajaba en el proceso de liberalización de la vida familiar, social y en general asistimos al ocaso del Auxilio Social, a partir de los sesenta (Vilanou \& Planella, 2010, p. 168) y las escuelas hogar (Servicio Social de la Mujer). Juan XXIII se refería al nuevo escenario sociológico intentando despertar a la Iglesia, de ahí su intervención en el Congreso de Estudio sobre la Mujer y la Sociedad. Todo ello sin renunciar al sentido tradicional de familia cristiana, una referencia de primer orden en los esquemas más tradicionalistas del nacionalcatolicismo. El núcleo familiar está asociado a un esquema ideológico (control social, idea del corporativismo totalitario, la intervención de la religión).

\section{Conclusiones}

1. Las mentalidades no cambian tan rápidamente, la idea del Estado paternalista y protector, con la colaboración de la Iglesia, persiste hasta el final del Franquismo. No obstante, la influencia del ambiente en la obra educativa de la Iglesia transformó el espíritu de la acción educativa, una acción que se fue haciendo cada vez más social y menos asistencial. En el marco que las instituciones educativas católicas han creado florecen nuevos proyectos e iniciativas que en muchos casos huyen de la rutina y la superficialidad que imponen los grupos hegemónicos de la sociedad y de la propia Iglesia. Los agentes de la acción educativa popular cristiana no tienen la visibilidad que siguen manteniendo en España quienes están posicionados en espacios de poder. En consecuencia, su historia debe ser recuperada y su impacto investigado con rigor, sin prejuicios.

2. Hasta finales de los cincuenta, buena parte de los proyectos católicos de corte popular pivotaban sobre el mundo rural y las clases urbanas más desfavorecidas, en el marco de la caridad y la propaganda católica. En las congregaciones religiosas y en los movimientos laicales, sobre todo, la pedagogía cobró un protagonismo de primer orden. Favorecidos por el contexto de cambio económico, sectores católicos influidos por Illich y Paulo Freire, a la luz del diagnóstico de sociólogos y de otros profesionales, por una parte, y en el ámbito del compromiso personal y social llegan a las clases populares gracias a las apuestas patronales y el modelo empresarial educativo que apuesta por la educación para ganar el futuro. Sin la colaboración de la burguesía católica y la utopía de la generación de sacerdotes, religiosos y religiosas jóvenes difícilmente puede explicarse el cambio de paso del modelo educativo entre los cristianos españoles (BrunoJofré, 2017).

3. Durante las décadas de 1950 y 1960 el tejido educativo del catolicismo se fue transformando en su interior, como consecuencia del cambio de posición de muchos efectivos católicos que se desmarcaron del modelo de educación de cristiandad, que había crecido gracias al modelo escolar del nacionalcatolicismo. El cambio más relevante durante los sesenta se expresa en el paso de un modelo educativo propio del catolicismo social y de los congresos católicos del ochocientos, a un universo de compromiso social, basado en una nueva lectura de la realidad social y de la incardinación de sacerdotes, religiosos, religiosas y lacios en las periferias del Estado. Sectores católicos comprometidos con la justicia social, se mostraron especialmente activos e innovadores en el terreno de la escolarización y la educación social, y se profesionalizaron, al punto de situarse entre las vanguardias del cambio político. 


\section{Referencias bibliográficas}

Amat, J. (2017). Com una pàtria. Barcelona: Edicions 62.

Armengou, M.; Belis, R. (2016). Els internats de la por. Barcelona: Ara Llibres.

Barciela López, C. (2000). La ayuda americana en España (1953-1963). Alicante: Universidad de Alicante.

Bruno-Jofré, R. (2016). Las religiosas del Niño Jesús en Bembibre, León, España, en la segunda etapa del franquismo: la escuela sin puertas. Historia de la Educación. Revista Interuniversitaria, 35, 53-70.

Cañabate, J. A. (2003-2004). La pugna entre la Iglesia Católica y el Frente de Juventudes en el ámbito educativo. Referencias internacionales, antecedentes y trayectoria general durante el primer franquismo. Historia de la Educación. Revista Interuniversitaria, 22-23, $105-$ 121.

Carmona, F. J. (2016). Autocrítica del catolicismo español. Sociología religiosa y acción pastoral. In F. Montero Garcia; J. Louzao, J. (eds.). Catolicismo y Franquismo. En la España de los años cincuenta. Autocríticas y convergencias (pp. 53-69). Granada: Comares Historia.

Comín, A. C. (1973). Educación 1938-1970. De la ideología espiritualista a la ideología tecnocrática. Cuadernos para el Diálogo, extra XXXVII, noviembre.

Corrales, X. (2008). De la misa al tajo. La experiencia de los curas obreros. Valencia: Universitat de València.

Dard, S. (2001). La educación popular católica en Barcelona durante la Restauración: de la beneficencia a la 'cuestión social'. Historia de la Educación. Revista Interuniversitaria, 20, 67-93.

Dávila Balsera, P. (2011). Las órdenes y congregaciones religiosas francesas y su impacto sobre la educación en España. Siglos XIX y XX. In J. M. Hernández Díaz (ed.). Francia en la educación de la España contemporánea (1808-2008) (pp. 101-159). Salamanca: Ediciones de la Universidad de Salamanca.

De Andrés Creus, L. (2013). Barraques. La lluita dels invisibles. Barcelona: Ara Llibres.

De Eguillor, Ma. J.; Vilanova, M. (2010). El riesgo de la utopía. Memoria de María José Sirera Olig (1934-1982). Barcelona: Universidad de Barcelona.

Delgado Gómez-Escamilla, L.; Martín de la Guardia, R.; Pardo Sanz, R. (coord.) (2017). La apertura internacional de España. Entre el franquismo y la democracia, 1953-1986. Madrid: Sílex.

Forcades, T. (2015). Por amor a la justicia. Doroty Day y Simone Weil. Madrid: Ediciones HOAC.

Fullana Puigserver, P. (2011). Historia de la Congregación de las Hijas de la Misericordia. Vol II: 1921-1968. Palma de Mallorca: Lleonard Muntaner Editor.

Fullana, P.; Montero, F. (2003-2004). Los modelos educativos juveniles del movimiento católico en España (1868-1968). Historia de la Educación. Revista Universitaria, 22-23, 33-51.

Fullana, P.; Ostolaza, M. (2007). Escuela católica y modernización. Las nuevas congregaciones religiosas en España (1900-1930). In J. de la Cueva; F. Montero (eds.). La secularización conflictiva. España (1898-1931) (pp. 187-213). Madrid: Biblioteca Nueva.

Galí, A. (1986). Història de les institucions i del moviment cultural a Catalunya, 1900-1936. Llibre XX: Col·laboració de l'Església al moviment cultural de Catalunya durant el segle $X X$. Barcelona: Fundació Alexandre Galí. 
Grup de Recerca d'Etnohistòria del Barraquisme (2008). El barraquisme a la ciutat de Barcelona. Can Valero, La Perona i el Carmel. Revista d'Etnologia de Catalunya, 33, 170-180.

Guereña, J. L.; Tiana Ferrer, A. (2016). Formas y espacios de educación popular en la Europa Mediterránea. Siglos XIX-XX. Madrid: Casa Velázquez-UNED Editorial.

Hermet, G. (1980). Los católicos en la España franquista. I. Los actores del juego político. Madrid: Centro de Investigaciones Sociológicas.

Hernández Díaz, J. M. (2011). Francia en la educación de la España contemporánea (18082008). Salamanca: Ediciones de la Universidad de Salamanca.

Hernández Burgos, C. (2013). Franquismo a ras de suelo. Zonas grises, apoyos sociales y actitudes durante la dictadura (1936-1976). Granada: Ediciones Universidad Granada.

Holgado, J.; Márquez, J. M.; Martín, M. (2013). De la Escuela de Magisterio de la Iglesia al CES Cardenal Spínola CEU (1957-2011). Historia de una institución. Sevilla: CEU Fundación San Pablo Andalucía.

Fullana Puigserver, P. (2005). Pere Capellà (1907-1954). La lluita incansable per la llibertat. Algaida: Ajuntament d'Algaida.

Fullana Puigserver, P.; Ostolaza Esnal, M. (2007). Escuela católica y modernización. Las nuevas congregaciones religiosas en España (1900-1930). In J. de la Cueva; F. Montero (eds.). La secularización conflictiva. España (1898-1931) (pp. 187-213). Madrid: Biblioteca Nueva.

Fullana Puigserver, P. (2015). Parroquias misioneras en Puente Vallecas: un espacio de diálogo entre el catolicismo y la clase obrera 1939-1953. In F. Montero; J. Louzao (eds.), La restauración social católica en el primer franquismo, 1939-1953 (pp. 115-136). Alcalá de Henares: Universidad de Alcalá.

Illich, I. (1971). La sociedad desescolarizada. Barcelona: Seix Barral.

Judt, T. (2008). Sobre el olvidado siglo XX. Madrid: Taurus.

Kung, H. (2017). Siete papas. Experiencia personal y balance de la época. Madrid: Trotta Editorial.

Laffite, M. (1964). La mujer en España. Cien años de historia (1860-1960). Madrid: Editorial Aguilar.

Lamet, P. M. (2013). Azul y Rojo. P. José María Llanos. Biografía del jesuita que militó en las dos Españas y optó por el suburbio. Madrid: La Esfera de los libros.

López Noguero, F. (2004). La educación Popular en España. Retos e interrogantes. Ágora Digital, 7, 1-13.

Mata, M. (1966). El tema de l'educació cristiana. Qüestions de Vida Cristiana, 33-34, 137-144.

Matas Pastor, J. J. (ed.) (2015). Las Hermanas de la Pureza de María en la formación de maestros y comunicadores. Historia del Centro de Enseñanza Superior Alberta Giménez (1974-2014). Madrid: Universidad Pontificia de Comillas - CESAG.

Matas Pastor, J. J. (2015a). Crònica 50 anys. Mater Misericordiae. Palma de Mallorca: Rapitbook.

Monés, J. (1981). L'escola a Catalunya sota el franquisme. Barcelona: Edicions 62.

Montero, F. (1988). Propaganda católica y educación popular en la España de la Restauración. In École et Église en Espagne et en Amérique Latine. Actes du Colloque de Tours (4-6 décembre 1987) (pp. 265-279). Tours: Publications de l'Université de Tours.

Montero, F. (2001). El catolicismo social durante el Franquismo. Sociedad y Utopía. Revista de Ciencias Sociales, 17, 115-134. 
Montero, F. (2007). Las derechas y el catolicismo español: del integrismo al socialcristianismo. Historia y Política, 18, 101-128.

Montero, F. (2016). La Acción Católica especializada y la educación popular. Las campañas de los movimientos católicos juveniles durante el Franquismo“. In J. L. Guereña; A. Tiana Ferrer (eds.). Formas y espacios de educación popular en la Europa Mediterránea. Siglos $X I X-X X$ (pp. 331-358). Madrid: Casa Velázquez-UNED Editorial.

Montero, F. (2017). El Movimiento Católico en España, 1889-1936. Alcalá de Henares: Universidad de Alcalá de Henares.

Montero, F.; Louzao, J. (coord.) (2015). La restauración social católica en el primer franquismo, 1939-1953. Alcalá de Henares: Universidad de Alcalá de Henares.

Montero, F.; Louzao, J. (eds.) (2016). Catolicismo y franquismo en la España de los años cincuenta. Autocríticas y convergencias. Granada: Comares Historia.

Moreno Seco, M. (2005). Mujeres, clericalismo y asociacionismo católico. In J. de la Cueva Merino; A. L. López Villaverde (coord.). Clericalismo y asociacionismo católico en España: de la restauración a la transición (pp. 107-131). Cuenca: Ediciones de la Universidad de Castilla la Mancha.

Muñoz Soros, J. (2016). La política educativa y cultural de Joaquín Ruiz-Giménez (1951-1956). In F. Montero; J. Louzao (eds.). Catolicismo y franquismo en la España de los años cincuenta. Autocríticas y convergencias (pp. 123-151). Granada: Comares Historia.

Nuq, A. (2012). La rééducations des jeunes déviants dans les maisons de redressement de l'Espagne franquiste (1939-1979). Aix: Université Aix-Marseille.

Ostolaza Esnal, M. (2000). Entre Religión y Modernidad. Los colegios de las congregaciones religiosas en la construcción de la sociedad guipuzcoana contemporánea, 1876-1931. Bilbao: Universidad del País Vasco. Servicio Editorial.

Palacio Lis, I.; Ruiz Rodrigo, C. (2010). L'educació social a Espanya durant el Franquisme. In C. Vilanou; C. Planella (eds.). De la compassió a la ciutadania (pp. 163-188). Barcelona: Editorial UOC.

Preiswerk, M. (1994). Educación popular y teología de la liberación. San José de Costa Rica: DEI.

Prellezo, J. M. (2012). Las escuelas profesionales salesianas. Momentos de su historia. Madrid: Editorial CCS.

Raguer, H. (2001). El quadern de Monjuic: records de la vaga de tramvies. Barcelona: Claret.

Raguer, H. (2006). Réquiem por la Cristiandad. El Concilio Vaticiano II y su impacto en España. Barcelona: Península.

Regás, R. (2004). El pozo del miedo. In E. Silva; A. Esteban; J. Castán; P. Salvador (coord.). La memoria de los olvidados. Un debate sobre el silencio de la represión franquista. (pp. 69-74). Valladolid: Ámbito.

Rodríguez Leal, S. (2007). La iglesia en Vallecas. Del Padre Llanos a Enrique de Castro, 19551987. Revista de Dialectología y Tradiciones Populares, 62/1, 205-247.

Rodríguez Leal, S. (2017). Alberto, ciudadano vallecano. In E. Robles (ed.). Alberto Iniesta, la caricia de Dios en las periferias (pp. 59-82). Barcelona: Herder.

Roig López, O. (2002). La institución educativa española desde la postguerra hasta la transición. Iglesia y tecnología. Barcelona: UAB.

Sainz Martínez, J. C. (1996). De FECUM a FECUM: política y religión entre los Congregantes Marianos (1965-1977). Política y Sociedad, 22, 103-121. 
Sánchez Andrés, A. (1969). El Patronato de la Juventud Obrera de Valencia. Saitabi, 19, 1969, 185-200.

Sanz Fernández, F. (2001). La Juventud Obrera Cristiana: un movimiento educativo popular. Historia de la Educación. Revista Interuniversitaria, 20, 2001, 95-115.

Townson, N. (coord.) (2009). España en cambio. El segundo Franquismo, 1959-1975. Madrid: Siglo XXI.

Viñao, A. (2004). Escuela para todos. Educación y modernidad en la España del siglo XX. Madrid: Marcial Pons.

Yetano, A. (1988). La enseñanza religiosa en la España de la Restauración (1900-1920). Barcelona: Anthropos. 